\title{
Gravitating Disks Around Black Holes
}

\section{Vladimír Karas and Ladislav Šubr}

\author{
Astronomical Institute, Academy of Sciences, Prague, Czech Republic \\ Email: vladimir.karas@cuni.cz
}

\begin{abstract}
Fluid disks and tori around black holes are discussed within different approaches and with the emphasis on the role of disk gravity. We first review the prospects for investigating the gravitational field of a black hole-disk system by analytical solutions of stationary, axially symmetric Einstein equations. More detailed considerations are focused on the middle and outer parts of extended disk-like configurations where relativistic effects are small and the Newtonian description is adequate. As an example, we investigate the case of a torus near a massive black hole that is a member of the black-hole binary system.
\end{abstract}

Keywords. accretion, accretion disks, gravitation, black hole physics

Almost three decades ago, Wilson (1981) speculated about the importance of accretion disk self-gravity in cataclysmic binaries. In this case, the role of self-gravity was rejected on the basis of detailed arguments. However, more recently Karas et al. (2004) applied a similar set-up to disks surrounding the primary component of a supermassive black hole binary. We examined the rich structure of Roche lobe surfaces (see Figure 1) and studied the regions where instabilities are expected due to self-gravity.
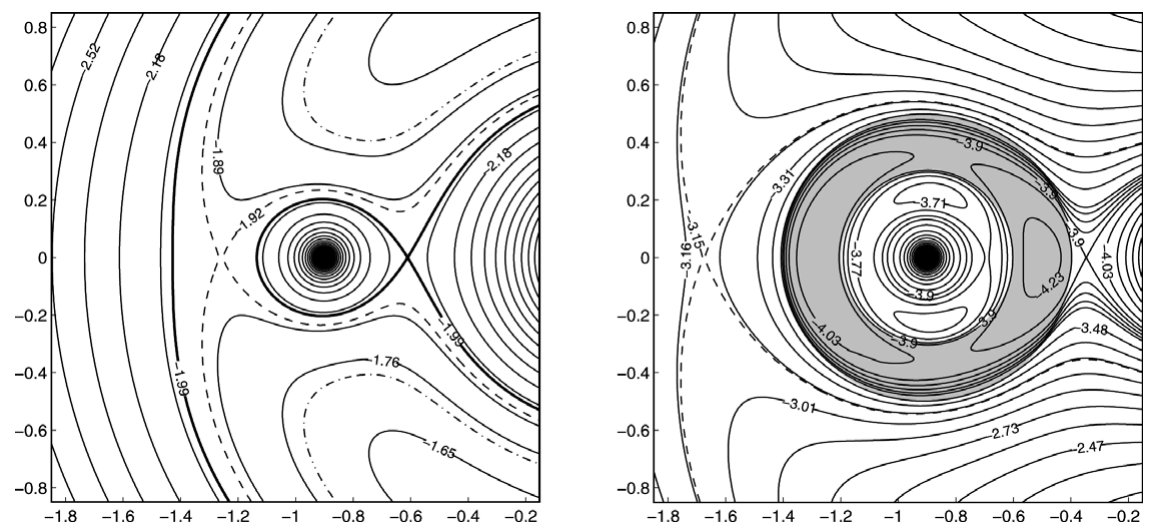

Figure 1. Left: Traditional contours of the Roche potential are plotted for two point masses, representing supermassive black holes in their orbital plane (mass ratio 0.1). Right: As on the left, but with a contribution due to a massive disk (shaded) taken into account as an additional source of the gravitational field. The plot shows the potential structure in the critical region near the cusp where the mass exchange takes place.

\section{References}

Karas, V., Huré, J.-M., \& Semerák, O. 2004, Classical and Quantum Gravity, 21, R1

Wilson, R. E. 1981, ApJ, 251, 246 